\title{
Diseño universal de aprendizaje en la enseñanza de la Física
}

\author{
Ana R. Barrón ${ }^{1}$ y Mario H. Ramírez ${ }^{\star}$ \\ (1) Instituto Politécnico Nacional, Centro de Investigación en Ciencia Aplicada y Tecnología Avanzada, Unidad Legaria, \\ Calzada Legaria 694, Ciudad de México, México (correo-e: anabarronhdz@hotmail.com). \\ (2) Instituto Politécnico Nacional, Centro de Investigación en Ciencia Aplicada y Tecnología Avanzada, Unidad Legaria, \\ Calzada Legaria 694, Ciudad de México, México (correo-e: mramirezd@ipn.mx).
}

${ }^{*}$ Autor a quien debe ser dirigida la correspondencia.

Recibido May. 15, 2021; Aceptado Jul. 13, 2021; Versión final Ago. 16, 2021, Publicado Dic. 2021

\begin{abstract}
Resumen
El propósito de este estudio es adaptar el Diseño Universal de Aprendizaje (DUA) a la enseñanza de la física a estudiantes de educación secundaria. En este estudio, el maestro desarrolla una diferenciación específica y oportuna de sus lecciones tomando en cuenta el DUA como punto de partida para planificar la enseñanza, flexibilizar los componentes, y alcanzar las metas propuestas. Los resultados muestran que la metodología no solo potencia una enseñanza efectiva y novedosa, sino que también permite un grado de adaptación diferenciado que puede aplicarse a variedad de necesidades y requerimientos. Se muestra que la enseñanza por parte de los maestros puede ofrecer una variedad de ambientes de aprendizaje universales que ofrecen múltiples oportunidades, pero con adaptaciones específicas. Se concluye que la atención a la diversidad en las aulas debe ser atendida por la autoridad educativa a través de la participación de cada uno de los actores educativos y que la responsabilidad no solo debe recaer en los docentes.
\end{abstract}

Palabras clave: física educativa; diseño universal; aprendizaje; estrategias; representaciones

\section{Universal design for learning in the teaching of Physics}

\begin{abstract}
The primary goal of this study is to adapt the Universal Design for Learning (UDL) methodology for the teaching of physics to secondary school students. In the present study, teachers develop a differential and timely differentiation for their lessons by applying the UDL methodology as the starting point for planning lessons, adapting teaching components, and achieving their set goals. The results show that the UDL methodology not only enhances effective and innovative teaching, but it also allows a different degree of adaptation that can be applied to a variety of needs and requirements. This allows teachers to provide a variety of universal learning environments that offer multiple opportunities, but with specific adaptations. It is concluded that enhancing classroom diversity is a duty that must be addressed by educational authorities through active participation of each of the components of the educational system and that this should not be left to teachers only.
\end{abstract}

Keywords: physics education; universal design; learning; strategy; representation 


\section{INTRODUCCIÓN}

"El mundo de la educación está experimentando actualmente una transformación masiva como resultado de la revolución digital" (Collins y Halverson, 2009), por lo que es importante y recomendable, utilizar esta tecnología para la mejora del currículum y programa de enseñanza de la ciencia; como menciona Wehmeyer et. Al. (2008) la tecnología tiene el potencial de contribuir a una mejor calidad de vida para los estudiantes que enfrentan alguna discapacidad o barrera para el aprendizaje, que es más que una simple cuestión de conveniencia. Entendamos barrera para el aprendizaje, en los términos que hoy en día se utilizan, como lo señala la Secretaría de Educación Pública (SEP, 2016) aquellas situaciones de aprendizaje que representan una barrera y que hacen referencia a todas las dificultades que experimentan los alumnos al interactuar entre ellos y entre todos los elementos del contexto educativo (las personas, las políticas, las instituciones, las culturas y las circunstancias sociales y económicas que afectan sus vidas).

"Durante la última década, la enseñanza y el aprendizaje en las instituciones de educación... se ha visto profundamente influenciado por la adopción generalizada de Internet y las tecnologías de comunicación en red, y, el uso estratégico y el aprendizaje mejorado por la tecnología (TEL)" (Dinmore, 2014), así como nuevas tecnologías para la comunicación y la información (NTIC's), Tecnología de asistencia (Johnston, et. al. 2007), etc.; posibilitan una oportunidad única para la implementación de enfoques de diseño universal debido a que enriquecen el entorno de aprendizaje mejorado por la tecnología. Utilizar la Tecnología para favorecer la inclusión a través del diseño universal de aprendizaje presenta obstáculos, (Mull y Sitlington 2003) hay 5 obstáculos identificados para la implementación: 1) el uso de tecnología de asistencia en el papel de 'prótesis cognitiva', 2) la disponibilidad y el alto costo de la tecnología de asistencia, 3) abandono por parte de los estudiantes de dispositivos de tecnología de asistencia comprados, 4) necesidades de capacitación relacionadas con el uso de tecnología y dispositivos de asistencia, y 5) preguntas de elegibilidad; por lo que de acuerdo a dichos autores, es necesario I. identificar el dispositivo adecuado, II. Evaluar las necesidades de los estudiantes y III. Capacitar a los estudiantes en su uso adecuado (Mull y Sitlington 2003). "Los educadores de ciencias deben estar equipados con ideas, herramientas y el apoyo necesario para crear espacios seguros e inclusivos para el aprendizaje colaborativo" (Baurhoo y Asghar, 2014).

"Planificar la inclusión educativa es una tarea difícil, dado que desde los profesores, la educación es un proceso imprevisible y la diversidad estudiantil es cada vez mayor, siendo el tiempo no lectivo un recurso insuficiente para favorecer la inclusión en las escuelas" (Escobar, 2020). Además, "la formación docente en el marco de la educación inclusiva debe responder a los fundamentos teóricos tales como las discapacidades y condiciones excepcionales" (Román y Meléndez, 2021), la adaptación de la tecnología de asistencia adecuada para estudiantes que presentan barreras para el aprendizaje puede posibilitar la mejora del logro educativo mediante la creación de oportunidades y espacios que maximicen su potencial, seguridad e independencia utilizando materiales de bajo costo y elaborando tareas que sean capaces de realizar. Por otro lado, la enseñanza de las ciencias puede y debe utilizar estas herramientas, pero no sólo centrarse en ellas. Recordemos que la ciencia y su enseñanza, en la actualidad sigue asociándose al uso exclusivo y forzoso de las NTIC's, olvidando que en la antigüedad, los conocimientos trascendentales que poseemos hoy en día fueron consolidados sin el uso de los formatos digitales o remotos que utilizamos hoy en día.

En México, existen indicadores que representan una preocupación para la mejora de la enseñanza, de acuerdo a los nuevos enfoques educativos a nivel mundial, tal como lo señala Flores (2012), uno de tales indicadores es "la formación recibida por los docentes para enseñar ciencias de manera eficiente, es incompleta", es frecuente que estas disciplinas se apeguen al dictado y memorización de información, generando una idea distorsionada del ejercicio de la ciencia. Garduño (2019) también señala que "la ciencia ha estado al margen tanto de la formación de los maestros como de su vida cotidiana", este arquetipo suele reproducirse, cuando el docente entra al aula de clase y "lleva a cabo experimentos en los que se siguen ciertos pasos mecánicos con el fin de obtener un resultado establecido, impidiendo un verdadero aprendizaje con significado" (Garduño, 2019), de esta forma se transmite y perpetua el modelo.

Por otra parte, ha habido cambios aplicados en la enseñanza de la ciencias en el país, en 1997 la Secretaría de Educación Pública puso en marcha los proyectos EFIT (física) y EFAT (matemáticas); "EI modelo EFIT es el resultado de la adaptación a la enseñanza de la física en la Escuela Secundaria Mexicana del modelo canadiense Technology Enhanced Science Secondary Instruction (tessi)" (Flores-Camacho, 2012). Dicho programa confluyen tecnologías digitales y elementos básicos de laboratorio de física, se utilizaron computadoras, juegos de sensores (temperatura, voltaje, sonido, distancia y fuerza), software de simulación, videos, equipo de laboratorio, etc. Sin embargo, como señala el INEE (Instituto Nacional para la Evaluación en Educación en México, en un estudio de las estrategias utilizadas en la enseñanza de la ciencia, "Los resultados arrojados por el estudio longitudinal realizado en la aplicación de todos estos modelos muestran que los procesos de apropiación del uso de la tecnología por parte de los maestros son lentos y una apropiación adecuada depende fuertemente de un uso frecuente e intensivo de la tecnología y de una capacitación continua realizada por expertos" (Flores-Camacho, 2012). 
La Física ha sido considerada una de las ciencias "duras" y de difícil comprensión para la mayoría de los estudiantes; además, hay un rechazo generalizado hacia el contenido de la materia, por la naturaleza conceptual de la misma y su relación con los conceptos matemáticos que permiten su decodificación; así mismo, "la ciencia no forma parte de nuestra cultura, lo cual se traduce en la manera como la sociedad mexicana la concibe y considera que debe enseñarse" (Garduño, 2019). Además, de estas situaciones, el docente debe enfrentarse a llevar a cabo su práctica con grupos numerosos, llegando a tener 300 o más alumnos, y ahora la Secretaría de Educación Pública de México (SEP) con la nueva "Escuela mexicana" considerar a los alumnos que enfrentan barreras para el aprendizaje dentro del grupo regular, "la tarea es eliminar las barreras para el aprendizaje y la participación (BAP)" (SEP, 2018); por lo que el ejercicio de la docencia, hoy por hoy, representa un gran reto para los docentes de física, que no sólo deben salir avante con las circunstancias del contexto y de su propia formación, sino que deben prepararse para adaptar su enseñanza en aras de la inclusión.

En otro contexto, las nuevas tecnologías de la información representan un nuevo marco de enseñanza, bajo el cual, las ciencias han tomado fuerza para responder a los retos específicos del área, "la educación tiene la exigencia de formar estudiantes que sean competentes dentro de una sociedad que gira en torno a la información y se nutre de las herramientas que facilitan procesos de comunicación más efectivos y con menos barreras" (Castinlanco, Vizcaino, 2008); además de que representan una enseñanza informal, con la cual los estudiantes conviven en su día a día y se informan; como se señala en el WEF (World Economic Forum) 2016 (citado en Melo et. al. 2017) "no son solamente una necesidad en la sociedad actual, sino que lideran la transición hacia la cuarta revolución industrial". La enseñanza de la física, como señalan Castinlanco, Vizcaino, 2008, a través de las NTICS representa la oportunidad de: a) Fortalecer la capacidad de abstracción y raciocinio; b) Trabajar sobre los procesos de pensamiento, creatividad y de análisis frente a la producción, uso y asimilación de las NTICS; c) Ejercicios fundamentados en los avances científicos de la Física, y otros inspirados en las necesidades que impone la ciencia para mejorar procesos de investigación; y d) Desarrollar la inteligencia tecnológica, (Reflexionar, producir, adaptar) para fortalecer la inteligencia científica (analizar, reflexionar, construir). Por todo esto, la enseñanza de la física a través de las NTICS representa una oportunidad de ajustar la enseñanza a las nuevas necesidades del estudiante del siglo XXI, una oportunidad de desarrollar capacidades que permitan la inserción al mundo actual.

Las NTIC's se piensan como objetos digitales o tecnologías virtuales, que requieren de una interfaz o conectarse a una red, olvidando que algunas herramientas son elaboradas de manera manual o artesanal; el uso de material de laboratorio y sensores está clasificado dentro de esta categoría de enseñanza, así como juegos o juguetes analógicos que son utilizados de manera didáctica. Hay docentes quienes elaboran sus propios recursos, que les permiten darle versatilidad y adaptabilidad a su práctica carente de tecnologías de punta. Ante esto, surge la pregunta ¿De qué manera podemos implementar las NTIC's a nuestra práctica? y ¿qué herramientas son adecuadas para mi contexto o situación? Este trabajo pretende responde a tal pregunta.

En la actualidad, ha cobrado fuerza a lo largo del mundo un modelo de enseñanza El Diseño Universal del Aprendizaje (por sus siglas en español DUA) que considera fundamental el diseño y planificación universal desde un cero, "ofrece orientación en el desarrollo y mantenimiento de entornos físicos y de aprendizaje accesibles para todos los estudiantes" (Dalton, E et. Al.2019:1); este movimiento se originó desde hace ya varios años, en Estados Unidos, y fue creado por el Centro de Tecnología Especial y Aplicada (CAST, 2018); sus fundadores el Neuropsicólogo David H. Rose y la Doctora en Educación Anne Meyer. Como señala Alba et. al. $(2011,2015)$ el DUA, Se fundamentó de los aprendizajes obtenidos de múltiples esferas del conocimiento, como la arquitectura y el urbanismo. Sus principios marcaron la pauta en el diseño y creación de espacios, objetos, y producciones que posibilitaran entornos flexibles y sostenibles, donde cualquier tipo de persona pudiera desenvolverse, sin necesidad de adecuación o adaptación ; por poner un ejemplo, "las adaptaciones a un edificio que beneficiarían solo a la población con discapacidad, conforme fue creciendo el movimiento y se hacían las modificaciones, se pudo corroborar que también hubo consecuencias positivas para la población sin discapacidad, ya que todos usaban tales diseños"(SEP, 2018).

Más tarde, el movimiento trascendería al ámbito educativo, teniendo gran aceptación por la comunidad docente y por los padres y niños que urgían un cambio en la forma en la que se venía enseñando décadas atrás; ya que el UDL consideraba desde un principio, un diseño o planificación global, donde todo tipo de estudiantes encajaban, "El marco del DUA estimula la creación de diseños flexibles desde el principio, que presenten opciones personalizables que permitan a todos los estudiantes progresar desde donde ellos están y no desde donde nosotros imaginamos que están» (CAST, 2011). Ahora bien, hasta este punto podría confundirse los términos universalidad con uniformidad, debido a que ambos conceptos tienen connotaciones similares, pero no iguales. El modelo DUA tiene claro la diferencia, mientras que uniformidad puede definirse como semejante o igual, universalidad es "que pertenece o se extiende a todos, sin excepción de ninguno" (RAE, 2020); en educación la igualdad es importante, refiriéndonos a derechos y obligaciones, pero en aprendizaje es fundamental establecer una equidad, entre las necesidades de los estudiantes, la cual puede garantizarse si se trabaja desde un enfoque universal; igualdad de derechos, pero equidad en oportunidades de aprendizaje, 
ofrecer los mismas situaciones didácticas para todos los estudiantes, pero desde diferentes escenarios que permita a todos acceder al conocimiento.

\section{OTROS ANTECEDENTES}

Hay una serie de antecedentes adicionales que es necesario detallar para documentar en mejor forma este trabajo: i) Diseño de materiales y actividades didácticas; y ii) El panorama en la Física.

\section{Diseño de materiales y actividades didácticas}

De acuerdo con la SEP (2018) "el DUA se entiende como el diseño de materiales y actividades didácticas que permiten que los objetivos de aprendizaje sean alcanzados por individuos con amplias diferencias en sus capacidades" (SEP, 2018). Se fundamenta en las teorías del constructivismo de L. Vygotsky, con su teoría de la construcción social del aprendizaje; y la teoría de las inteligencias múltiples de Gardner; para establecer una serie de directrices que se relacionen con las competencias o saberes (SEP, 2018). Se ha basado en tres principios: a. Múltiples formas de representación: el qué del aprendizaje; b. Múltiples formas de acción y expresión: el cómo del aprendizaje; c. Múltiples formas de motivación: el porqué del aprendizaje. Estos principios se traducirán en pautas de acción que concuerdan con tareas específicas de los dos hemisferios cerebrales, especialmente aquellas regiones especializadas en el tratamiento e interpretación de la realidad, es decir, diversas interpretaciones de la realidad; y además que se relacionen con la Física y se concreten en acciones reales y plausibles. "Prácticas de enseñanza inclusivas donde no es necesario separar los contenidos, o planear diferente y menos separar por grupos de discapacidad o alumnos regulares, si no que las didácticas pedagógicas de aula se planean y ejecutan en tal dinamismo que haya lugar a los principios mencionados y por lo tanto los participantes puedan participar de acuerdo a sus capacidades y fortalezas" (Figueroa et. al 2019).

Hay estudiantes que reconocen y procesan la información de mejor manera usando medios audiovisuales; otros aplican análisis, diseño y creación de estrategias para resolver un problema, pero no son diestros en su ejecución como otros podrían hacerlo; otros estudiantes son hábiles con ensayo y error, lo que les da la capacidad de aprender sobre la marcha; y otros tantos requieren de ejemplos específicos que les permita orientar su propio criterio. Independientemente de esto, debemos mantener presente siempre, el papel sustancial que juega la motivación que el docente imprima, y la comprensión de las necesidades específicas y apoyos de los estudiantes; por citar la un ejemplo de aplicación de una tecnología a la educación, "el éxito de los estudiantes con discapacidades que participan en las clases de STEM de educación general se relaciona directamente con las habilidades de los maestros para comprender las necesidades de aprendizaje únicas de los estudiantes y sus habilidades para resolver problemas (Marino, 2010:1), ya que hay estudiantes que, si no son estimulados apropiadamente con recursos y materiales de apoyo adecuados y pertinentes, simplemente la estrategia o actividades pasarán desapercibidas. "El docente siempre será el mediador entre un currículo incluyente y otro excluyente, será la dinámica creativa de una pedagogía en movimiento la que establezca los límites de la inclusión" (Figueroa, et. al, 2019) ya que la mayoría necesita apoyos y / o andamios para convertirse en aprendices expertos, que el docente puede ayudar a lograr.

Tomando en cuenta los principios mencionados, y la literatura analizada se establecen "un conjunto de sugerencias concretas para aplicar el marco UDL, practicar y ayudar a garantizar que todos los alumnos puedan acceder y participar en oportunidades de aprendizaje significativas y desafiantes" (CAST, 2018), las cuales se muestran en la tabla 1. Los cuatro componentes principales de DUA son: Las metas, los métodos, los materiales y la evaluación (CAST 2018 y Ralabate 2011) "Las metas se describen típicamente como expectativas de aprendizaje, Los métodos son estrategias de instrucción utilizadas por los educadores para apoyar el aprendizaje de los estudiantes, los materiales son los medios que se utilizan para presentar el contenido y demostrar el aprendizaje, la evaluación se refiere al proceso de recopilar información sobre el progreso de un alumno utilizando una variedad de métodos y materiales". Del dominio e implementación efectiva que el docente de a estos elementos dependerá el éxito de aprendizaje de los estudiantes ya que el propósito "es crear estudiantes expertos: en la evaluación e identificación de sus propias necesidades, monitorear su propio progreso, regular y mantener su interés, esfuerzo y persistencia durante una tarea de aprendizaje" (Ralabate, 2011).

\section{El panorama en la Física}

Educar desde la universalidad implica no solo afrontar tales retos, sino aspirar a conseguir una colaboración que no conozca límites, sea flexible y siempre tienda a la mejora, avanzando en términos de inclusión y justicia. Así mismo, la educación es una tarea que implica la participación de todos los actores sociales, los maestros, directivos, y padres de familia. Aunque parezca una tarea difícil, es cierto que la Física no está exenta de considerar todos estos múltiples factores para proveer un panorama que sea beneficioso para todos los estudiantes y que permita cambiar la perspectiva que se ha tenido sobre la materia. Algunos puntos fundamentales sobre el proceso de enseñanza-aprendizaje en física que el docente debe regularmente analizar y evaluar sobre la marcha se muestran en la tabla 2. 
Tabla 1: Sugerencias para aplicar el marco UDL.

\begin{tabular}{|c|c|c|}
\hline $\begin{array}{l}\text { I. Proporcionar múltiples formas de re- } \\
\text { presentación }\end{array}$ & $\begin{array}{l}\text { II. Proporcionar múltiples formas de } \\
\text { acción y expresión }\end{array}$ & $\begin{array}{l}\text { III. Proporcionar múltiples formas } \\
\text { de implicación }\end{array}$ \\
\hline $\begin{array}{l}\text { 1. Proporcionar diferentes opciones } \\
\text { para percibir la información }\end{array}$ & $\begin{array}{l}\text { 4. Proporcionar múltiples medios físi- } \\
\text { cos de acción }\end{array}$ & $\begin{array}{l}\text { 7. Proporcionar opciones para cap- } \\
\text { tar el interés }\end{array}$ \\
\hline $\begin{array}{l}\text { 1.1. Opciones que permitan modificar } \\
\text { y personalizar la presentación de la in- } \\
\text { formación }\end{array}$ & $\begin{array}{l}\text { 4.1. Proporcionar varios métodos de } \\
\text { respuesta }\end{array}$ & $\begin{array}{l}\text { 7.1. Optimizar la elección individual } \\
\text { y la autonomía }\end{array}$ \\
\hline $\begin{array}{l}\text { 1.2. Ofrecer alternativas para la infor- } \\
\text { mación auditiva }\end{array}$ & $\begin{array}{l}\text { 4.2. Ofrecer diferentes posibilidades } \\
\text { para interactuar con los materiales }\end{array}$ & $\begin{array}{l}\text { 7.2. Optimizar la relevancia, el valor } \\
\text { y la autenticidad }\end{array}$ \\
\hline $\begin{array}{l}\text { 1.3. Ofrecer alternativas para la infor- } \\
\text { mación visual }\end{array}$ & $\begin{array}{l}\text { 4.3. Integrar el acceso a herramientas } \\
\text { y tecnologías de asistencia }\end{array}$ & $\begin{array}{l}\text { 7.3. Minimizar la sensación de inse- } \\
\text { guridad y las distracciones }\end{array}$ \\
\hline $\begin{array}{l}\text { 2. Proporcionar múltiples opciones } \\
\text { para el lenguaje y los símbolos }\end{array}$ & $\begin{array}{l}\text { 4.3. Integrar el acceso a herramientas } \\
\text { y tecnologías de asistencia }\end{array}$ & $\begin{array}{l}\text { 8. Proporcionar opciones para man- } \\
\text { tener el esfuerzo y la persistencia }\end{array}$ \\
\hline $\begin{array}{l}\text { 2.1. Definir el vocabulario y los símbo- } \\
\text { los }\end{array}$ & $\begin{array}{l}\text { 5.1. Utilizar múltiples formas o medios } \\
\text { de comunicación }\end{array}$ & $\begin{array}{l}\text { 8.1. Resaltar la relevancia de las } \\
\text { metas y los objetivos }\end{array}$ \\
\hline 2.2. Clarificar la sintaxis y la estructura & $\begin{array}{l}\text { 5.2. Usar múltiples herramientas para } \\
\text { la composición y la construcción }\end{array}$ & $\begin{array}{l}\text { 8.2. Variar los niveles de desafío y } \\
\text { apoyo }\end{array}$ \\
\hline $\begin{array}{l}\text { 2.3. Facilitar la decodificación de tex- } \\
\text { tos, notaciones matemáticas y símbo- } \\
\text { los }\end{array}$ & $\begin{array}{l}\text { 5.3. Incorporar niveles graduados de } \\
\text { apoyo en los procesos de aprendizaje }\end{array}$ & $\begin{array}{l}\text { 8.3. Fomentar la colaboración y la } \\
\text { comunidad }\end{array}$ \\
\hline $\begin{array}{l}\text { 2.4. Promover la comprensión entre di- } \\
\text { ferentes idiomas }\end{array}$ & $\begin{array}{l}\text { 6. Proporcionar opciones para las fun- } \\
\text { ciones ejecutivas }\end{array}$ & $\begin{array}{l}\text { 8.4. Proporcionar una retroalimen- } \\
\text { tación orientada }\end{array}$ \\
\hline $\begin{array}{l}\text { 2.5. Ilustrar las ideas principales a } \\
\text { través de múltiples medios }\end{array}$ & $\begin{array}{l}\text { 6.1. Guiar el establecimiento de metas } \\
\text { adecuadas }\end{array}$ & $\begin{array}{l}\text { 9. Proporcionar opciones para la } \\
\text { autorregulación }\end{array}$ \\
\hline $\begin{array}{l}\text { 3. Proporcionar opciones para la com- } \\
\text { prensión }\end{array}$ & $\begin{array}{l}\text { 6.2. Apoyar la planificación y el desa- } \\
\text { rrollo de estrategias }\end{array}$ & $\begin{array}{l}\text { 9.1. Promover expectativas y } \\
\text { creencias que optimicen la motiva- } \\
\text { ción }\end{array}$ \\
\hline 3.1. Activar los conocimientos previos & $\begin{array}{l}\text { 6.3. Facilitar la gestión de información } \\
\text { y de recursos }\end{array}$ & $\begin{array}{l}\text { 9.2. Facilitar niveles graduados de } \\
\text { apoyo para imitar habilidades y es- } \\
\text { trategias }\end{array}$ \\
\hline $\begin{array}{l}\text { 3.2. Destacar patrones, características } \\
\text { fundamentales, ideas principales y re- } \\
\text { laciones entre ellas }\end{array}$ & $\begin{array}{l}\text { 6.4. Mejorar la capacidad para hacer } \\
\text { un seguimiento de los avances }\end{array}$ & $\begin{array}{l}\text { 9.3. Desarrollar la autoevaluación y } \\
\text { la reflexión }\end{array}$ \\
\hline \multicolumn{3}{|l|}{$\begin{array}{l}\text { 3.3. Guiar el procesamiento de la infor- } \\
\text { mación, la visualización y la manipula- } \\
\text { ción }\end{array}$} \\
\hline $\begin{array}{l}\text { 3.4. Maximizar la memoria y la transfe- } \\
\text { rencia de información }\end{array}$ & & \\
\hline
\end{tabular}

Es altamente probable que todos los docentes en algún momento de nuestra práctica hayamos incorporado aspectos del DUA, pero puede haber experiencias que nos hayan representado un problema para dar una respuesta pertinente a una barrera del estudiante o que ni si quiera hayamos podido percibir. El DUA ofrece una estructura sistemática y completa para dar solución a estos posibles inconvenientes que no solo beneficiarán a los estudiantes regulares, sino que de manera universal impactará en todos, de diversas formas y gradualidad; "la aplicación del enfoque del diseño universal en el proceso educativo, en general, y en el ámbito universitario, en particular, benefician tanto a estudiantes como a profesores y, por tanto, se configura como un paradigma válido cuando el objetivo es mejorar las condiciones de enseñanza y aprendizaje de todas las personas" (Sánchez, Díez, Martín, 2016).

Ahora bien, tomando en cuenta todos lo elementos anteriormente descritos, es vital esclarecer el enfoque que nuestra propuesta plantea implementar desde la física, y los ajustes que debemos realizar al estándar del aprendizaje para volverlo universal "Los estándares académicos proporcionan un punto de referencia para las habilidades y los conocimientos esenciales que los estudiantes deben dominar a medida que avanzan en los niveles de grado. Los maestros pueden identificar metas que están alineadas con los estándares académicos y proporcionar varias formas de lograr las metas, integrando estrategias de instrucción que sirven como apoyo y andamiaje para ayudar a todos los estudiantes a progresar hacia el dominio de las lecciones basadas en estándares" (Rao, y Meo 2016). 
Tabla 2: Puntos fundamentales para considerar en la física, desde el modelo de DUA.

\begin{tabular}{|l|l|}
\hline Comunicación constante & $\begin{array}{l}\text { Comunicar los objetivos y aprendizajes esperados de la lección así como los productos que } \\
\text { se espera realicen }\end{array}$ \\
\hline Flexibilidad en las tareas & $\begin{array}{l}\text { Ejemplificar las posibles tareas y sus adecuaciones que puedan realizar, en función de sus } \\
\text { necesidades e intereses }\end{array}$ \\
\hline Modalidades de trabajo & $\begin{array}{l}\text { Permitir la libre elección de modalidades de trabajo, tales como el trabajo individual, en } \\
\text { equipo, los lugares dentro del aula, así como algunos recursos como los audífonos que les } \\
\text { permitan concentrarse }\end{array}$ \\
\hline Retroalimentación de calidad & $\begin{array}{l}\text { Brindar una actividad constante de feedback en las actividades, respondiendo a todas las } \\
\text { posibles dudas que surjan durante el desarrollo de las lecciones }\end{array}$ \\
\hline $\begin{array}{l}\text { Diversidad de los recursos y } \\
\text { materiales }\end{array}$ & $\begin{array}{l}\text { Ofrecer los espacios, recursos y materiales tales como las NTIC's, STEAM, ODA's y otras } \\
\text { tecnologías de apoyo que les ofrezcan oportunidades desafiantes y motivantes }\end{array}$ \\
\hline Gestión del aprendizaje & $\begin{array}{l}\text { Desarrollo de tareas mediante la autodisciplinas control meticuloso del propio avance y mo- } \\
\text { nitoreo oportuna para los ajustes razonables. }\end{array}$ \\
\hline Funciones ejecutivas & $\begin{array}{l}\text { Fomentar las habilidades cognitivas complejas (planificar, organizar, guiar, revisar, regulari- } \\
\text { zar y evaluar), partiendo del fortalecimiento de las habilidades mentales básicas (memoria, } \\
\text { atención, lenguaje, razonamiento, etc). }\end{array}$ \\
\hline Educación emocional & $\begin{array}{l}\text { Potenciar el desarrollo de las habilidades emocionales, a través de la motivación y tutoría } \\
\text { oportuna (búsqueda del bienestar personal y social). }\end{array}$ \\
\hline
\end{tabular}

El propósito del estudio hecho en este documento es que el maestro pueda desarrollar una diferenciación específica y oportuna de las lecciones, tomando en cuenta el DUA como punto de partida para la planificación de su enseñanza, flexibilizando los componentes y alcanzando las metas propuestas; "al considerar el DUA durante el proceso de planificación y agregar vías flexibles para alcanzar las metas de aprendizaje, los maestros pueden reducir las barreras que existen en los planes de estudio y aumentar las oportunidades que permiten que todos los estudiantes alcancen los mismos altos estándares" (Rao y Meo 2016). El modelo permite visualizar los elementos principales (metas, método, materiales, evaluación) y más, así como los principios y pautas del DUA necesarios para que pueden integrarlo a su planificación de diferentes formas. Los maestros son libres de elegir los elementos que deseen, dada la realidad del aula y su propia iniciativa. Todos los indicadores no serán cubiertos de manera inmediata, pero algunos componentes sí, proporcionando poco a poco apoyos o andamios que beneficien a todos.

\section{METODOLOGÍA}

La investigación tiene un enfoque cualitativo, analizando dos estudios de caso. El objetivo principal es identificar los resultados de la aplicación de la propuesta metodológica de DUA en Física con dos profesores de contextos rural y urbano, los cuales imparten clase de Ciencias en Física en educación secundaria. La propuesta se ordena en 7 pasos, 1. Establecer metas o aprendizajes esperados (partiendo de los estándares e indicadores), 2. Identificar cuál es la problemática común para la comprensión del tema (hay estudios actuales sobre múltiples temas de la física con concepciones alternativas en común que puede ayudar a encontrar una solución o propuesta a su adecuado tratamiento; los anteriores pasos deben crear un ambiente de aprendizaje que posibilite la comprensión y el manejo de un lenguaje científico al alcance de los estudiantes; 3 . Elegir recursos de enseñanza-aprendizaje, los cuales deben responder a materiales, apoyos tecnológicos actuales o al manejo de juguetes físicos clásicos o tradicionales; 4. Fortalecer funciones ejecutivas, activación, enfoque, esfuerzo, emoción, memoria y acción, relacionándolos con las competencias en física; 5 . Mantener activada la motivación, el docente debe variar el método y las formas de trabajo que considere la variedad de estilos de aprendizaje (auditivo, visual, kinestésico, etc.) 6. Motivar los avances, a través del monitoreo constante del propio desempeño del alumno, apoyándolo a través del uso de las estrategias de autoaprendizaje; 7. Evaluación, Uso adecuado de materiales, evidencias, aplicaciones u otros recursos que le proporcionen información oportuna sobre su avance, logro y retos; los tres pasos anteriores, capacitando al estudiante en el monitoreo de su particular estilo de aprendizaje, sus avances y las estrategias ideales que le permitirán el cumplimiento de sus metas.

Estos siete pasos han sido ordenados de manera numérica, pero, no obedecen a una secuencia específica, sino que el docente puede ir alternando los pasos a como le sea conveniente, incluso omitir alguno o centrarse especialmente en uno de ellos. Pongamos un ejemplo (presentado en la Tabla 3), cada tema de física y sus indicadores requieren de un análisis detallado que permita entender cómo dosificar los contenidos, qué acciones tomar y bajo que procedimientos se llevará a cabo la planificación a través del modelo de DUA en Física; por lo que es importante considerarlo como un recurso flexible que puede ser modificado y que puede ser manipulable o perfeccionado. 


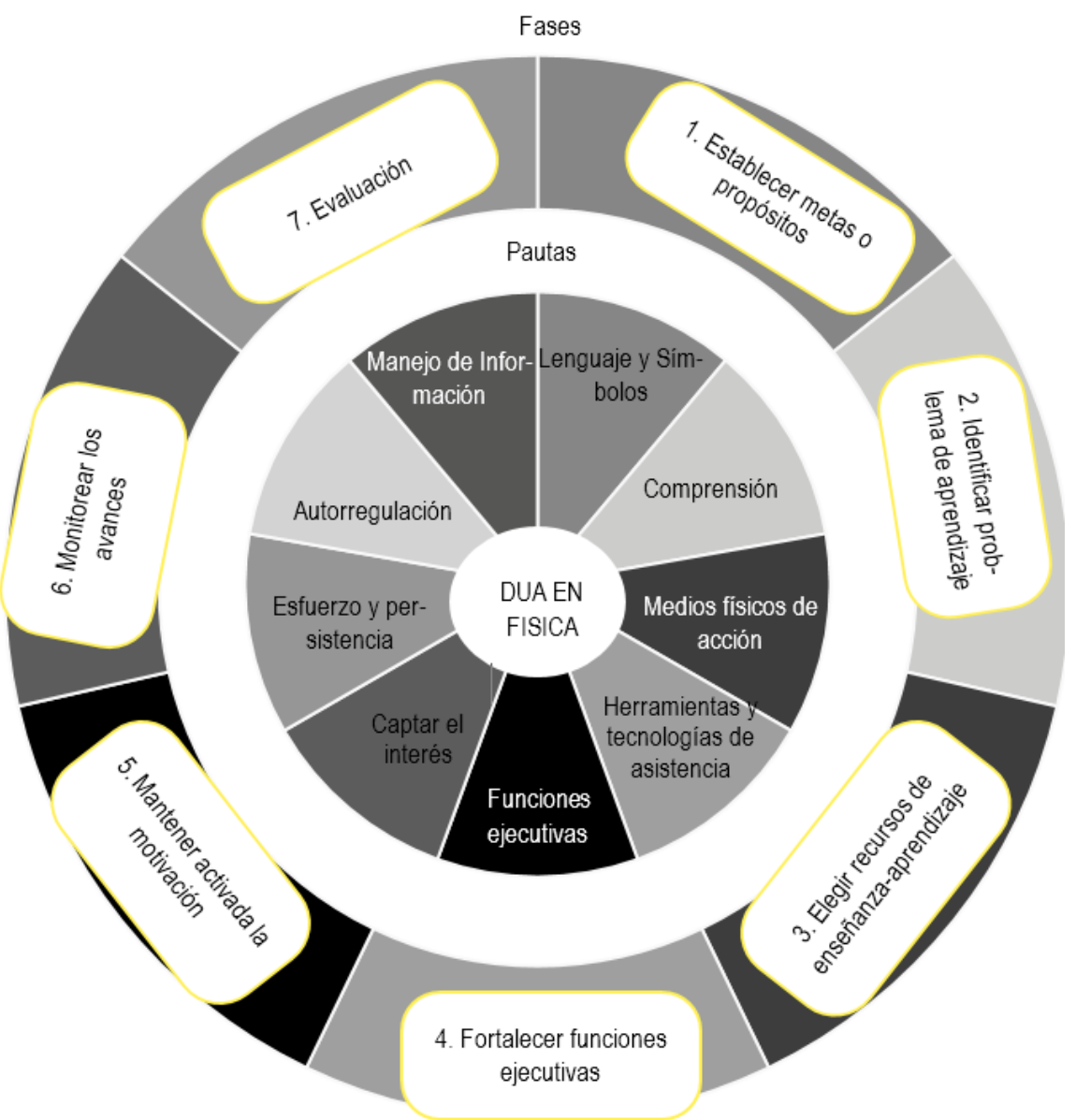

Fig.1: Modelo de DUA en Física.

Tabla 3. Ejemplo de pasos.

\begin{tabular}{|l|l|l|l|}
\hline $\begin{array}{l}\text { Paso 1. Establecer metas o } \\
\text { propósitos }\end{array}$ & $\begin{array}{l}\text { Paso 2. Identificar } \\
\text { problema de aprendizaje }\end{array}$ & $\begin{array}{l}\text { Paso 3. Elegir } \\
\text { recursos de E-A }\end{array}$ & $\begin{array}{l}\text { Paso 4. Fortalecer Funciones } \\
\text { Ejecutivas }\end{array}$ \\
\hline $\begin{array}{l}\text { Tema: Propiedades de la materia } \\
\text { Aprendizaje clave: }\end{array}$ & $\begin{array}{l}\text { Problema 1. Poca } \\
\text { claridad en el concepto } \\
\text { "materia" } \\
\text { modelo de partículas y comprende } \\
\text { su relevancia para representar la } \\
\text { estructura de la materia }\end{array}$ & $\begin{array}{l}\text { Problema 2. } \\
\text { Propiedades de la } \\
\text { materia no se analizan o } \\
\text { Meta: Reconocer el movimiento } \\
\text { cinético de las partículas de la } \\
\text { materia a través del estudio de su } \\
\text { comportamienton } \\
\text { Problema 3. No hay } \\
\text { relación del tema con la } \\
\text { realidad inmediata }\end{array}$ & $\begin{array}{l}\text { Juguetes caseros } \\
\text { Juegos dinámicos } \\
\text { Experimentos caseros y clase } \\
\text { demostrativas }\end{array}$ \\
\hline $\begin{array}{l}\text { Paso 5. Mantener activa la } \\
\text { motivación }\end{array}$ & $\begin{array}{l}\text { Paso 6. Monitorear los } \\
\text { avances }\end{array}$ & Paso 7. Evaluación & Observaciones \\
\hline $\begin{array}{l}\text { Trabajo en equipo } \\
\text { Mesa redonda (análisis de }\end{array}$ & $\begin{array}{l}\text { Uso de tablas de } \\
\text { categorización y tabla de } \\
\text { avances) }\end{array}$ & $\begin{array}{l}\text { Portafolio de } \\
\text { evidencias } \\
\text { Prueba rápida de 5 ó } \\
10 \text { reactivos } \\
\text { Exposición oral }\end{array}$ & $\begin{array}{l}\text { Los problemas encontrados } \\
\text { varios indicadores que } \\
\text { pueden fortalecerse a lo largo } \\
\text { de la lección y no solo de esta } \\
\text { meta en particular }\end{array}$ \\
\hline
\end{tabular}

\section{RESULTADOS}

Actualmente, el modelo ha sido aplicado en el nivel de educación secundaria en México, el cual corresponde al segundo nivel básico en el país con estudiantes entre 12-15 años. La implementación se basó en la asignatura de química, pero en un tema también de física; "Propiedades físicas de los materiales". A continuación en la Tabla 4, se presentan los pormenores de la implementación de la propuesta, detallando clase a clase y en la figura 2 se muestran algunas evidencias que la profesora colaboradora brindó para este estudio. Es interesante analizar que, en dicha propuesta, debido a la pandemia por Coronavirus, las clases no han podido ser presenciales, y han tenido que ser virtuales por lo que la docente ha tenido que ofrecer una variedad de adecuaciones que han transformado y limitado su práctica docente. 
Tabla 4. Puesta en acción. Clase 1

\begin{tabular}{|c|c|c|c|}
\hline $\begin{array}{l}\text { Paso 1. Establecer metas o propósi- } \\
\text { tos }\end{array}$ & $\begin{array}{l}\text { Paso 2. Identificar pro- } \\
\text { blema de aprendizaje }\end{array}$ & $\begin{array}{l}\text { Paso } 3 . \text { Elegir re- } \\
\text { cursos de E-A }\end{array}$ & $\begin{array}{l}\text { Paso 4. Fortalecer Funciones Eje- } \\
\text { cutivas }\end{array}$ \\
\hline \multirow[t]{4}{*}{$\begin{array}{l}\text { Tema: Identificación de las propieda- } \\
\text { des extensivas (masa y volumen) e } \\
\text { intensivas }\end{array}$} & $\begin{array}{l}\text { 1. No asocian el signifi- } \\
\text { cado literal de los términos } \\
\text { "Extensivo" e "Intensivo" } \\
\text { (por qué y simbología) }\end{array}$ & $\begin{array}{l}\text { Tablas de doble en- } \\
\text { trada }\end{array}$ & \multirow[t]{4}{*}{ Emoción y Esfuerzo } \\
\hline & $\begin{array}{l}\text { 2. No recuerdan o utilizan } \\
\text { las unidades de medición } \\
\text { de cada propiedad }\end{array}$ & $\begin{array}{l}\text { Presentador de Dia- } \\
\text { positivas }\end{array}$ & \\
\hline & & Plataforma virtual & \\
\hline & & $\begin{array}{l}\text { Cuestionario y Rú- } \\
\text { brica }\end{array}$ & \\
\hline $\begin{array}{l}\text { Paso 5. Mantener activa la motiva- } \\
\text { ción }\end{array}$ & $\begin{array}{l}\text { Paso 6. Monitorear los } \\
\text { avances }\end{array}$ & Paso 7. Evaluación & Observaciones \\
\hline \multirow{3}{*}{$\begin{array}{l}\text { Comunicación constante mediante } \\
\text { los espacios virtuales de aprendizaje } \\
\text { (plataforma) y aplicaciones de forta- } \\
\text { lecimiento conceptual "Kaahoot" }\end{array}$} & $\begin{array}{l}\text { Uso de plataforma Class- } \\
\text { room }\end{array}$ & Rúbrica & \multirow{3}{*}{$\begin{array}{l}\text { La educación es presencial pero } \\
\text { debido a la pandemia ha tenido } \\
\text { que buscarse estrategias para tra- } \\
\text { bajar de manera virtual }\end{array}$} \\
\hline & Chat en vivo o directo & $\begin{array}{l}\text { Fotografías de evi- } \\
\text { dencias }\end{array}$ & \\
\hline & Facebook y messenger & $\begin{array}{l}\text { Análisis de desem- } \\
\text { peño en plataforma } \\
\text { Prueba T y Google } \\
\text { Classroom }\end{array}$ & \\
\hline
\end{tabular}

\section{Puesta en acción. Clase 2}

En este caso, encontramos en la planificación (Tabla 5), elementos del modelo DUA en Física, que se enfocan en 1. Establecimiento de aprendizajes o propósitos, 2. Elegir recursos de aprendizaje, 3. Funciones ejecutivas, 4. Evaluación, 5. Mantener activada la motivación, además en la figura 3 se muestran algunas evidencias que la profesora colaboradora brindó para este estudio.

Tabla 5. Puesta en acción. Clase 2

\begin{tabular}{|l|l|l|l|}
\hline $\begin{array}{l}\text { Paso 1. Establecer metas o } \\
\text { propósitos }\end{array}$ & $\begin{array}{l}\text { Paso 2. Identificar problema } \\
\text { de aprendizaje }\end{array}$ & $\begin{array}{l}\text { Paso 3. Elegir } \\
\text { recursos de E-A }\end{array}$ & $\begin{array}{l}\text { Paso 4. Fortalecer Funciones } \\
\text { Ejecutivas }\end{array}$ \\
\hline $\begin{array}{l}\text { Tema: Identificación de las } \\
\text { propiedades físicas de los } \\
\text { materiales }\end{array}$ & $\begin{array}{l}\text { No hay claridad en el papel } \\
\text { que juega el acomodo de } \\
\text { las partículas atómicas de } \\
\text { cada elemento, no hay una } \\
\text { conexión entre lo que } \\
\text { acontece a nivel atómico } \\
\text { con el nivel } \\
\text { organismo/materia }\end{array}$ & $\begin{array}{l}\text { Simulador en línea } \\
\text { Hoja de texto } \\
\text { Plataforma virtual } \\
\text { Estructura de Lewis } \\
\text { y acomodo de } \\
\text { electrones }\end{array}$ & Acción y motivación \\
\hline $\begin{array}{l}\text { Paso 5. Mantener activa la } \\
\text { motivación }\end{array}$ & $\begin{array}{l}\text { Paso 6. Monitorear los } \\
\text { avances }\end{array}$ & Paso 7. Evaluación & Observaciones \\
\hline $\begin{array}{l}\text { Se utiliza un juego didáctico } \\
\text { tradicional, pero adaptado al } \\
\text { tema que estimule la } \\
\text { atención e interés }\end{array}$ & $\begin{array}{l}\text { Revisión vía windows y de } \\
\text { manera presencial }\end{array}$ & Rúbrica cualitativa & $\begin{array}{l}\text { Inicialmente los estudiantes no } \\
\text { conocían las reglas implícitas en el } \\
\text { acomodo de los electores, protones } \\
\text { yeutrones, sin embargo; } \\
\text { utilizando la información de la tabla } \\
\text { periódica se pudo establecer entre } \\
\text { todo el grupo las reglas del juego } \\
\text { que también se relacionan con la } \\
\text { teoría }\end{array}$ \\
\hline
\end{tabular}

A través de un juego tradicional (canicas) adaptado a las reglas del acomodo electrónico, la docente pudo establecer un puente entre la teoría y la práctica de una manera muy creativa que acaparó completamente la atención de los estudiantes, y en clases posteriores, analizó nuevamente el tema con ayuda de un simulador en línea. Aunque la docente refiere que únicamente consideró el paso no. 3 y 4 , para la planificación de la clase, podemos analizar que más elementos de DUA en Física han sido trastocados con esta estrategia utilizada. Además de que al analizar los resultados pudimos concluir dos problemas de aprendizaje que son comunes al estudiar el tema, que no se consideraron en la planificación, pero que inicialmente la maestra ya había considerado.

En ambas estrategias utilizadas por los docentes, podemos evidenciar que hay un trabajo de 5 de los 7 pasos propuestos en el modelo: 1. Establecer metas o aprendizajes esperados, 2. Identificar cuál es la problemática 
común para la comprensión del tema, 3. Elegir recursos de enseñanza-aprendizaje, 4. Fortalecer funciones ejecutivas, y 7. Evaluación; sin embargo, los pasos, 5. Mantener activada la motivación y 6 . Motivar los avances, no han sido fortalecidos en ambas propuestas.

\begin{tabular}{|c|c|c|c|}
\hline Fase & Partes de la planeación & ¿Qué se hace? & Taxonomia de Bloom \\
\hline \multirow[t]{2}{*}{ Inicio } & $\begin{array}{l}\text {-Objetivos de aprendizaje } \\
\text {-Enuncia las reglas de la } \\
\text { clase }\end{array}$ & $\begin{array}{l}\text { Explicación de las reglas del juego y la utilidad } \\
\text { Adecuación: el docente entrega o presenta de ma- } \\
\text { nera escrita las reglas del uuego, utilizando colores } \\
\text { y oraciones claras y precisas }\end{array}$ & $\begin{array}{l}\text { Recuerdo: Reconocer; Recordar; } \\
\text { Listar; Describir; Recuperar; Domi- } \\
\text { nar; y Localizar }\end{array}$ \\
\hline & $\begin{array}{l}\text {-Motivación } \\
\text {-Activación de conoci- } \\
\text { miento previo }\end{array}$ & $\begin{array}{l}\text { El estudiante retoma de sus evidencias de trabajo, } \\
\text { información sobre algunos elementos químicos y } \\
\text { las utiliza para elaborar un Diagrama de Bohr }\end{array}$ & $\begin{array}{l}\text { Comprensión: Interpretar; Ejempli- } \\
\text { ficar; Clasificar; y Resumir; } \\
\text { Inferir: Comparar; Explicar; y Para- } \\
\text { frasear }\end{array}$ \\
\hline Desarrollo & $\begin{array}{l}\text { Nuevo conocimiento } \\
\text { Modelación } \\
\text { Ejercitación }\end{array}$ & $\begin{array}{l}\text { El estudiante al finalizar el juego, deberá crear de } \\
\text { manera grupal o en equipos (organización que el } \\
\text { docente decida con sus alumnos) un instructivo de } \\
\text { las reglas del juego, ejemplificando con } 3 \text { elementos } \\
\text { El producto deberá ser ejemplificado con antelación } \\
\text { por el docente (tríptico, presentación de diapositi- } \\
\text { vas, etc.) } \\
\text { El estudiante define los términos clave como nú- } \\
\text { mero de protones y número de electrones y esta- } \\
\text { blece una representación simbólica } \\
\text { Adecuación: el estudiante con tdah podrá ser el en- } \\
\text { cargado de comprobar los ejemplos que el equipo } \\
\text { elija para ejemplificar, o podría realizar ejemplos en } \\
\text { formatos de coloreado similares al juego }\end{array}$ & 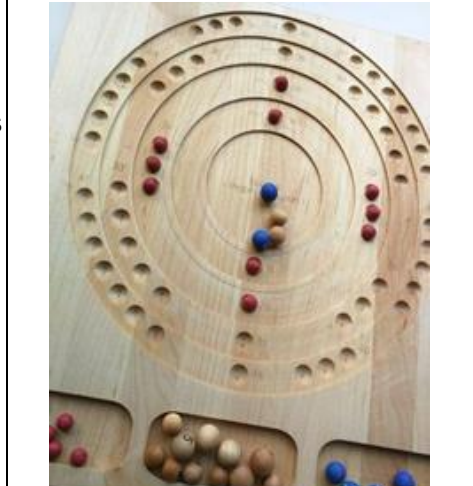 \\
\hline
\end{tabular}

Fig. 3: Evidencias de la Clase 2

La docente, aplica una estrategia lúdica, donde las reglas del juego no son explicadas por ella, sino que, mediante el trabajo en equipo, los estudiantes deberán descubrir o definir las reglas de operatividad del juego; durante la implementación de la estrategia estimula el trabajo cognitivo mediante la consolidación de los conceptos clave, a través de una actividad de clasificación. En un apartado de su planificación, la docente refiere una adecuación que tuvo que realizar al tener presente en su clase, estudiantes con TDAH. Con la finalidad de analizar el impacto del uso del modelo, se realizaron unos cuestionamientos a los docentes involucrados, quienes respondieron dos preguntas 1 . ¿Cuáles son las características del modelo que te beneficiarían al implementar en tu método de enseñanza? y ¿Cuáles fueron las dificultades que encontraste en su aplicación? Las respuestas a la primer pregunta fueron: 1) Es importante planificar teniendo en cuenta el problema de aprendizaje o los errores conceptuales comunes acerca de un tema; 2) Uso de Recursos y apoyos actuales, sean tecnológicos, analógicos o juegos tradicionales; y 3) La motivación y la educación emocional.

Muchos de los docentes planean sus clases sin tener en cuenta las necesidades conceptuales de los estudiantes o el nivel de expertis que poseen sobre un determinado tema; considerar las concepciones alternativas de los estudiantes, desde un inicio es una herramienta que nos puede traer múltiples beneficios, antes y después. En cuanto a los recursos o apoyos es predominante el uso de la pizarra o video, sin embargo pudiera considerarse poco pertinente ante la múltiple cantidad de tecnologías que son capaces de manejar los actuales estudiantes. Y la educación emocional, a pesar de estar ligada a asignaturas relacionadas a las ciencias sociales, recobra una importancia vital, al estar relacionada a la autorregulación del propio aprendizaje.

Por otro lado, las principales respuestas en común a la segunda interrogante fueron: 1) "La Figura Modelo de DUA en Física" no proporciona una explicación completa que describa claramente la operatividad del modelo; 2) El uso de Recursos o apoyos tecnológicos específicos representan un problema, al no tenerlos todos al alcance inmediato; 3) Temores sobre implementar una estrategia universal que detenga el desarrollo de los estudiantes regulares o avanzados; y 4) Necesitan capacitación para trabajar con los puntos 4, 5 y 6 del modelo. El concepto universalidad se asocia a igualdad, por lo que hay una opinión generalizada a que se dará las oportunidades desde un enfoque igualitario, se reducirá el nivel o eficacia de la enseñanza. Esto no es así, por lo que es importante ofrecer una variedad de estrategias que no permitan caer en lo monótono o comodidad al docente. 

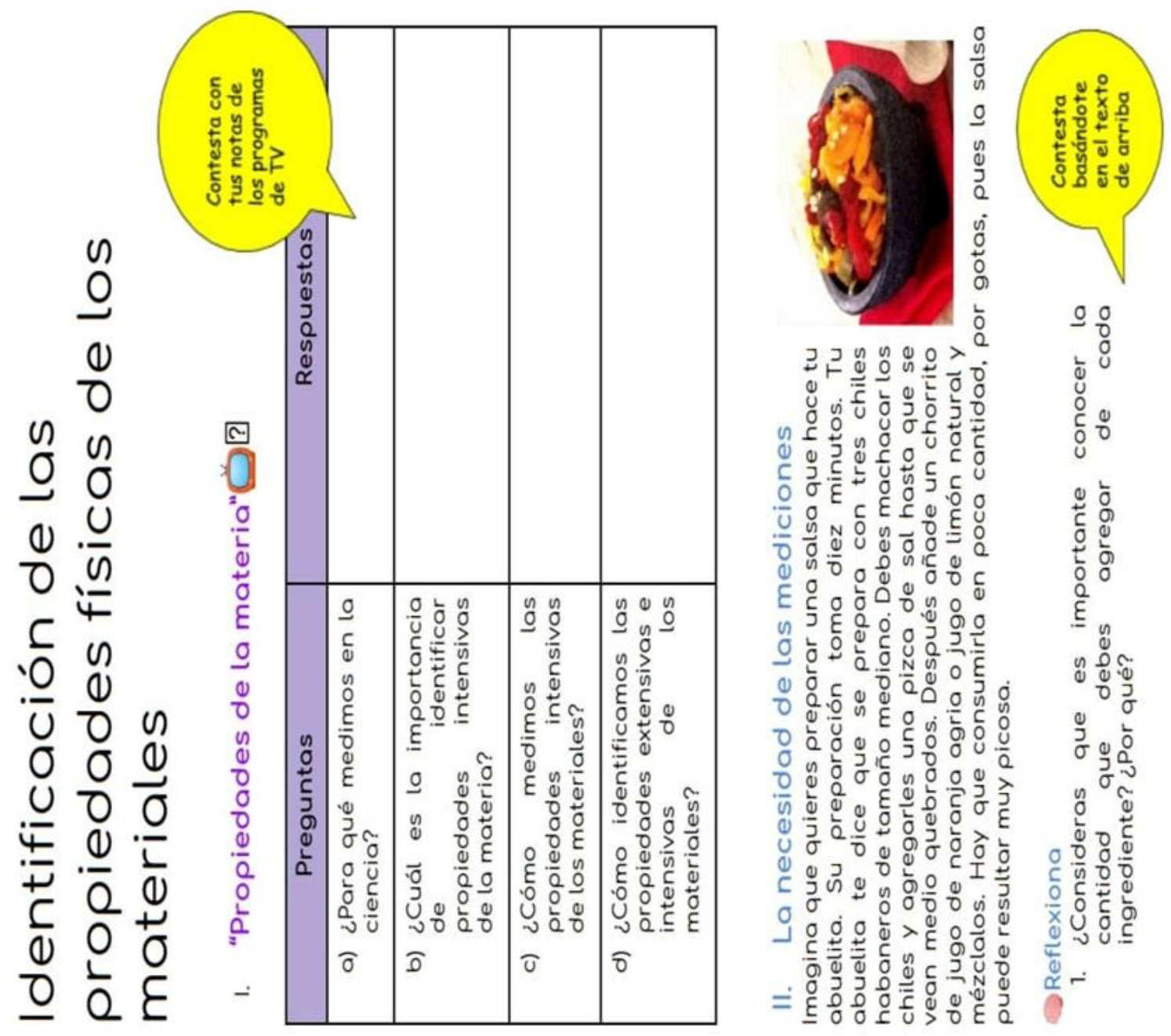

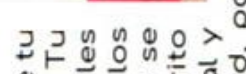

$\begin{array}{ll}0 & 0 \\ 0 & 0 \\ 1 & 0 \\ 0 & 0 \\ 0 \\ 0 \\ 0 & 0 \\ 0 & 0\end{array}$

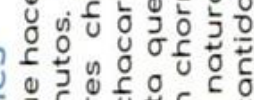

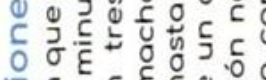

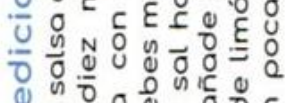

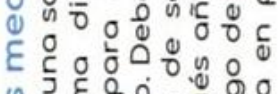

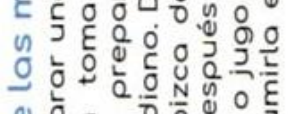

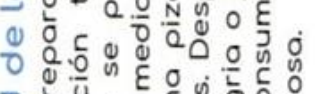

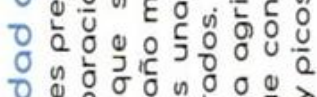

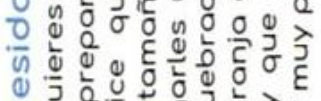

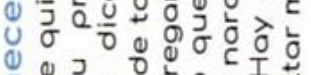

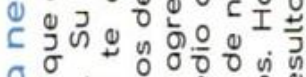

$\neg$ O

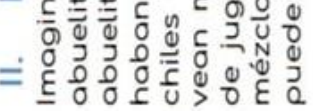
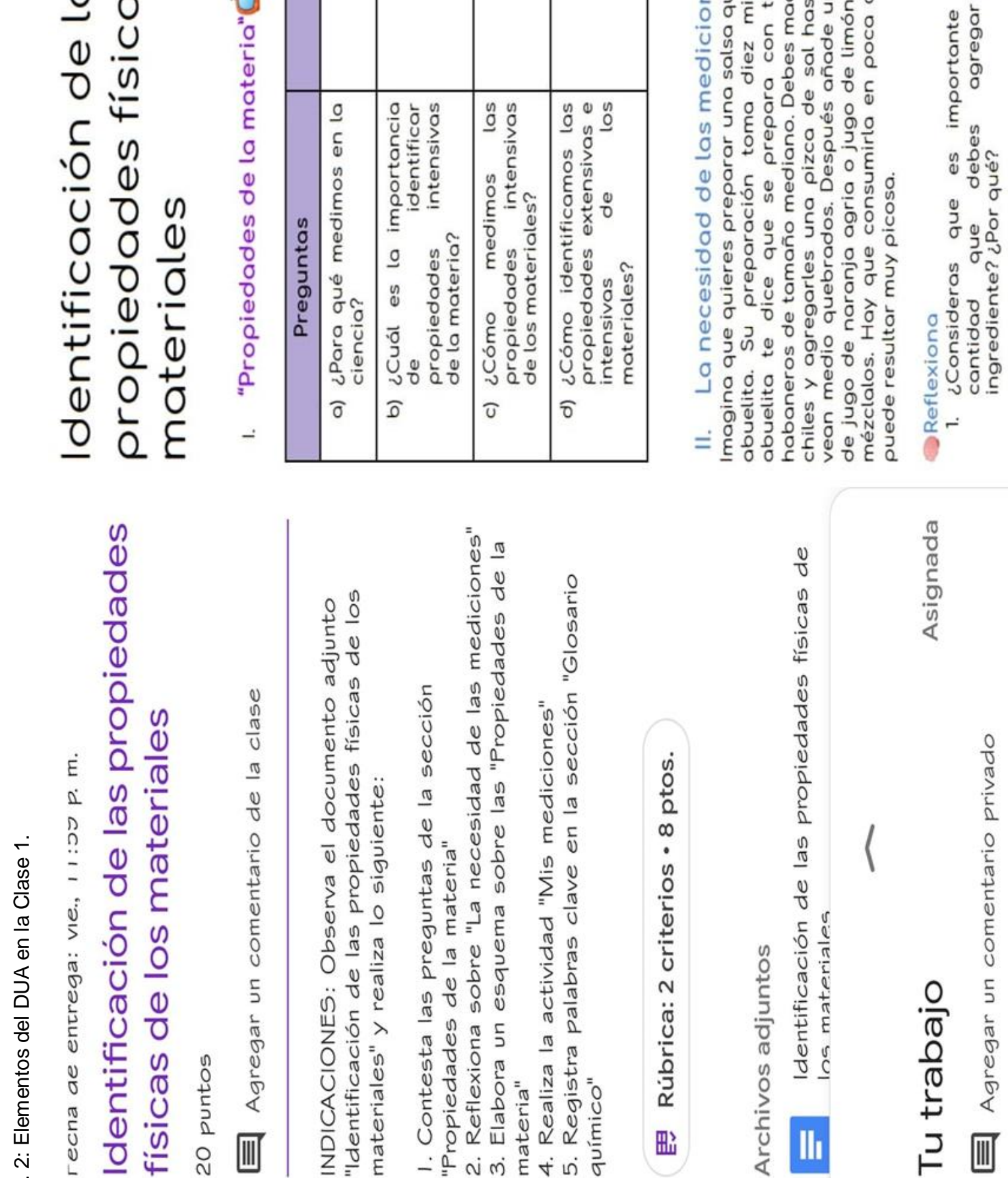

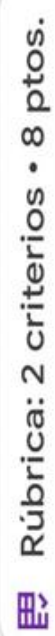

모 


\section{DISCUSION}

Es claro que es importante especificar cuáles son los elementos del modelo que lo hacen diferente de otras propuestas, uno de ellos es el "carácter de universalidad" que debe permitir ofrecer situaciones de aprendizaje que posibiliten, desde un inicio (la planificación o diagnóstico) la resolución de un conflicto, la implementación de una propuesta de impacto social, la incorporación de apoyos para personas discapacitadas o con barreras para el aprendizaje; el segundo elemento es la "inclusión" ya que partiendo desde este enfoque llegaremos al logro de una propuesta universal, que no separe, divide o distinga, sino que "Universalice" el conocimiento.

El docente, sea de Física o de otra asignatura, no debe esperar a encontrarse con problemas o desafíos para poder desarrollar oportunidades que preparen a los estudiantes. A través de los años, algunos docentes hemos desarrollado "adecuaciones" para aquellos alumnos que se rezagan, que se adelantan o que no pueden utilizar un material, como la mayoría de los alumnos regulares, pero aquí el cuestionamiento ¿Por qué no adelantarse? ¿Por qué no prevenir estas situaciones, planteándolas como una normalidad? ¿cómo una necesidad?

El modelo de DUA en física posibilita y acerca al docente a realizarse estos cuestionamientos, y a través de la consideración de los 7 pasos y el estudio a detalle de la manera de aplicar en el aula, nos acercará al logro de la "Universalización" en la enseñanza de la física. Ofreciendo una variedad de escenarios, desde los cuales, los estudiantes con diversos estilos de aprendizaje pueden trabajar en equipo y ayudarse unos a otros, complementándose y siendo protagonistas en distintos momentos, donde tomen el control y demuestren el dominio de un tipo de recurso o apoyo y ayuden a otros a adquirir tales habilidades.

Es difícil, que un docente logre ser experto en un determinado modelo de enseñanza, sobre todo cuando está acostumbrado a trabajar de una manera, y se rehúsa a abandonar las viejas prácticas y evita incluir nuevas; pero es importante destacar que el modelo DUA en Física, representa una oportunidad imperdible para dejar atrás la enseñanza tradicional y empezar a incluir experiencias que posibiliten la inclusión de todo tipo de aprendizajes a través de la universalización del ambiente de aprendizaje. Sin duda, el modelo DUA en Física, ofrecerá novedosos elementos a la práctica de los docentes.

\section{CONCLUSIONES}

Derivado del estudio realizado podemos concluir que 1. La atención a la diversidad en las aulas debe ser atendida por la autoridad educativa a través de la participación de cada uno de los actores educativos conforman (Supervisores, Directivos, Maestros, padres de familia, alumnos, etc.), la responsabilidad no solo debe recaer en los docentes, sino que debe haber un acompañamiento oportuno y específico que posibilite un ambiente inclusivo; 2. El docente, desde su trinchera, debe buscar el continuo mejoramiento de su práctica docente, se lo provea la autoridad educativa o no; en el mejoramiento de las prácticas educativas se encontrará el camino a la mejora y a la creación de ambientes inclusivos del aprendizaje; 3. El DUA en Física representa una oportunidad de mejora de la práctica docente, pero no una panacea que va a brindar de manera autómatica todas las adecuaciones o respuestas a las dudas que surjan sobre la marcha; hemos discutido las fortalezas y áreas de oportunidad que el modelo aquí propuesto produce, sin embargo creemos que son menos los puntos desfavorables y más los favorables; el DUA en Física considera los estilos y ritmos de aprendizaje, fomenta la educación emocional, la autorregulación del aprendizaje, el trabajo en equipo, la adecuación didáctica ante las barreras que se originan con el contexto, promueve el uso de las tecnologías en la enseñanza, pero también de la enseñanza tradicional a través de juegos, juguetes didácticos, experimentos, proyectos, canciones, etc., además deja abierta la posibilidad de modificar o mejorar aspectos del modelo a consideración personal del docente quien lo utilice. 4. Los docentes de Física, requieren fortalecer sus competencias en la enseñanza de la asignatura desde el enfoque universal de la enseñanza, considerando que podría existir una barrera para el aprendizaje o no; un docente que se apropia de este enfoque tenderá a considerar múltiples situaciones y mantendrá la intención de crear mejoras más allá de lo esperado, se podrá centrar en aspectos generales y que requieran especial atención, favorecerá la vinculación del aprendizaje con otras ciencias y disciplinas, será capaz de ver la enseñanza emocionalmente satisfactoria y transmitirla a sus estudiantes, porque desde la planificación ya estaría considerando que ocurrirá un problema con el aprendizaje y trabajará para resolverlo.

\section{REFERENCIAS}

Alba, C., Sánchez, J. y Zubillaga, A., Diseño universal para el aprendizaje: pautas para su introducción en el currículo, España, 1-45 (2015)

Alba, C., Sánchez, P., Sánchez, J. M., y Zubillaga, A., Pautas sobre el diseño universal para el aprendizaje (DUA), Universidad Complutense de Madrid, Revista Participación Educativa, (versión 2.0), 57- 66 (2011)

Alnahdi, G., Assistive Technology in Special Education and the Universal Design for Learning, Turkish Online Journal of Educational Technology - TOJET, 13, 18-23 (2014) 
Basham, J., y Marino, M.,, Understanding STEM education and supporting students through universal design for learning, https://doi.org/10.1177/004005991304500401,Teaching Exceptional Children, 45, 8-15 (2013)

Baurhoo N., y Asghar, A., Using Universal Design for learning to construct inclusive science classrooms for diverse lear, https://doi.org/10.36510/learnland.v7i2.651, Learning Landscapes Journal, 7, 74 (2014)

CAST., Universal design for learning guidelines version 2.2, The Center for Applied Special Technology website (2018)

CAST., Universal design for learning guidelines version 2.0 Traducción al español versión 2.0, The Center for Applied Special Technology website (2013)

CAST., Universal design for learning guidelines checklist, The Center for Applied Special Technology website (2019)

Castiblanco, O.L., y Vizcaíno, D.F., El uso de las TICs en la enseñanza de la física, Académico, 20-26 (2008)

Collins, A., y Halverson, R., Rethinking education in the age of technology, https://doi.org/10.1007/s11423-010-9159-

0, Journal of Computer Assisted Learning, 26,18-27 (2009)

Dalton, E.M., Lyner-Cleophas,M., Ferguson, B.T., y McKenzie, J., Inclusion, universal design and universal design for learning in higher education: South Africa and the United States, https://doi.org/10.4102/ajod.v8i0.519, African Journal of Disability, 8, 1-7 (2019)

Dinmore, S., The case for universal design for learning in technology enhanced environments,

https://doi.org/10.4018/ijcee.2014040103, International Journal of Cyber Ethics in Education, 3(2), 29-38 (2014)

Escobar, M., Muñoz, D., Piñones, C., y Cuadra, D., Tiempo escolar e inclusión educativa: un estudio de teorías subjetivas de profesores, 31(5), 139-152, https://doi.org/10.4067/s0718-07642020000500139 (2020)

Figueroa, L., Ospina, M., y Tuberquia, J., Prácticas pedagógicas inclusivas desde el diseño universal de aprendizaje y plan individual de ajuste razonable, https://doi.org/10.26620/uniminuto.inclusion.6.2.2019.4-14, Inclusión y Desarrollo, 6 (2) 4-14 (2019)

Flores-Camacho, F., La enseñanza de la ciencia en la educación básica en México, Otros textos de evaluación, 1aㅡ. Edición, México, INEE, 67-68 (2012)

Garduño, V., Mejorar la enseñanza de la ciencia, Revista de evaluación para docentes y directivos, Instituto Nacional de Evaluación Educativa (INEE), Blog Red (2019)

Johnston, L., Beard, L., y Carpenter, L., Assistive technology: access for all students, Columbus, OH, Prentice Hall (2007)

Rao, K., y Meo, G., Using universal design for learning to design standards-based lessons, https://doi.org/10.1177/2158244016680688, 1-12 (2016)

Marino, M. T., Defining a technology research agenda for elementary and secondary students with learning and other high incidence disabilities in inclusive science classrooms, https://doi.org/10.1177/016264341002500101, Journal of Special Education Technology 25, 1, 1-28 (2010)

Melo, D., Silva, J., Indacochea, L., y Núñez, J., Tecnologías en la educación superior: políticas públicas y apropiación social en su implementación, https://doi.org/10.19083/ridu.11.498, Revista Digital de Investigación en Docencia Universitaria, 11, 1, 193-206 (2017)

Mull, C., y Sitlington, P., The role of technology in the transition to postsecondary education of students with learning disabilities: a review of the literature, Journal of Special Education, 37, 26-32 (2003)

Ralabate, P., Universal design for learning: meeting the needs of all students, https://doi.org/10.1044/leader.FTR2.16102011.14, American Speech-Language-Hearing Association, 1-7 (2011)

RAE, Real Academia Española., Diccionario de la Lengua Española, 42.a ed., (2020)

Román, G., Pérez, E., Medina, A., Perfil del docente inclusivo de básica primaria: orientado a la transformación del proceso formativo, Información Tecnológica, 32(2), 89-108, http://dx.doi.org/10.4067/S0718-07642021000200089 (2021)

Sánchez, S., Díez, E., Martín, R., El diseño universal como medio para atender a la diversidad en la educación. Una revisión de casos de éxito en la universidad, https://doi.org/10.18172/con.2752, Contextos Educativos, 19, 121-131 (2016)

SEP, Secretaría de Educación Pública., Glosario de Educación Superior. México, 91 (2016)

SEP, Secretaría de Educación Pública., Buenas prácticas para la nueva escuela mexicana, inclusión; México, 1-4 (2018a)

SEP, Secretaría de Educación Pública., Aprendizajes clave para la educación integral: Estrategia de equidad e inclusión en la educación básica, México, 1-29 (2018b)

Wehmeyer, M., Palmer, S., Smith, S., Davies, D., Stock, S., The efficacy of technology use by people with intellectual disability: a single-subject design meta-analysis, https://doi.org/10.1177/016264340802300303, Journal of Special Education Technology, 23, 21-30 (2008) 